\title{
Asymptotic behaviour of a parabolic problem with terms concentrated in the boundary *
}

\author{
Ángela Jiménez-Casas ${ }^{1}$ \\ Aníbal Rodríguez-Bernal ${ }^{2}$
}

1 Grupo de Dinámica No Lineal. Universidad Pontificia Comillas de Madrid. C/Alberto Agulilera 23, 28015 Madrid.

2 Departamento de Matemática Aplicada. Universidad Complutense de Madrid, 28040 Madrid and Instituto de Ciencias Matemáticas, CSIC-UAM-UC3M-UCM.

\begin{abstract}
We analyze the asymptotic behavior of the attractors of a parabolic problem when some reaction and potential terms are concentrated in a neighborhood of a portion $\Gamma$ of the boundary and this neighborhood shrinks to $\Gamma$ as a parameter $\varepsilon$ goes to zero.
\end{abstract}

We prove that this family of attractors is upper continuous at $\varepsilon=0$.

\section{Introduction}

Let $\Omega$ be an open bounded smooth set in $\mathbb{R}^{N}$ with a $C^{2}$ boundary $\partial \Omega$. Let $\Gamma \subset \partial \Omega$ be a smooth subset of the boundary, isolated from the rest of the boundary, that is, $\operatorname{dist}(\Gamma, \partial \Omega \backslash \Gamma)>0$.

Define the strip of width $\varepsilon$ and base $\Gamma$ as

$$
\omega_{\varepsilon}=\{x-\sigma \vec{n}(x), x \in \Gamma, \sigma \in[0, \varepsilon)\}
$$

for sufficiently small $\varepsilon$, say $0 \leq \varepsilon \leq \varepsilon_{0}$, where $\vec{n}(x)$ denotes the outward normal vector. We note that for small $\varepsilon$, the set $\omega_{\varepsilon}$ is a neighborhood of $\Gamma$ in $\bar{\Omega}$, that collapses to the boundary when the parameter $\varepsilon$ goes to zero.

We are interested in the behavior, for small $\varepsilon$, of the solutions of the nonlinear parabolic problem

$$
\left(P_{\varepsilon}\right) \equiv\left\{\begin{array}{cc}
u_{t}^{\varepsilon}+A_{\varepsilon} u^{\varepsilon}+\mu u^{\varepsilon}=F_{\varepsilon}\left(x, u^{\varepsilon}\right) & \text { in }(0, T) \times \Omega \\
\frac{\partial u^{\varepsilon}}{\partial n}=0 & \text { on } \Gamma \\
u^{\varepsilon}(0)=u_{0} \in H^{1}(\Omega) &
\end{array}\right.
$$

*Partially suppported by Projects MTM2006-08262, CCG07-UCM/ESP-2393 UCM-CAM, Grupo de Investigación CADEDIF, PHB2006-003PC Spain and 775-UCIII, M.E.C. SPAIN. 


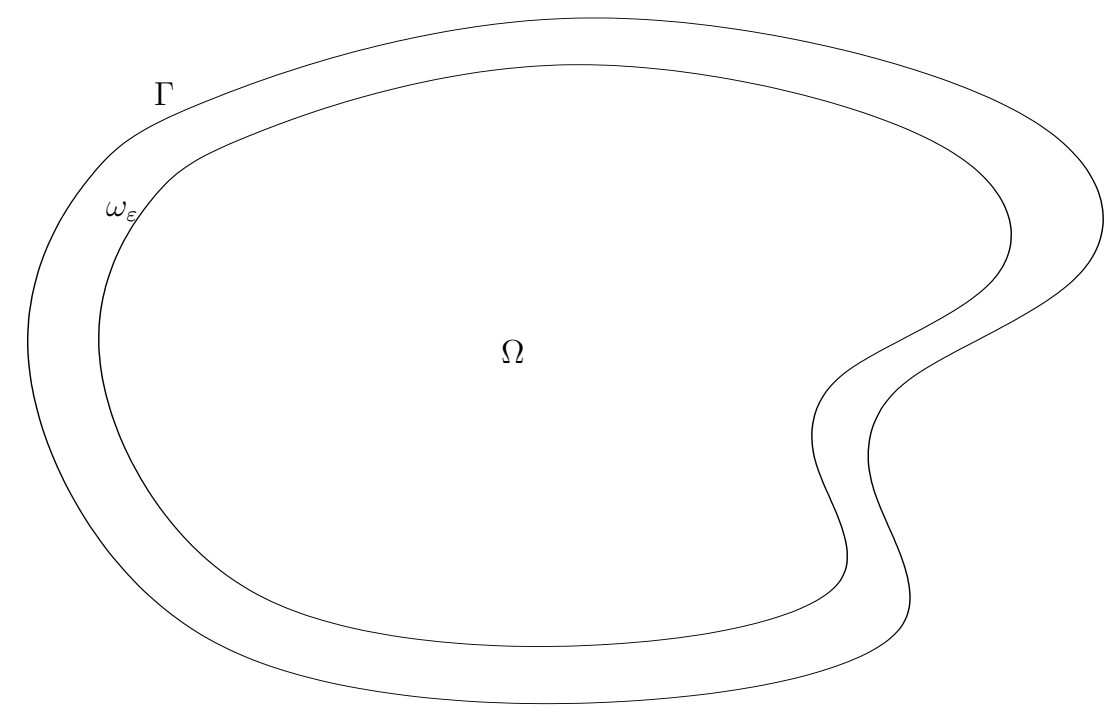

Figure 1: The set $\omega_{\varepsilon}$

where $A_{\varepsilon} u^{\varepsilon}=-\Delta u^{\varepsilon}+\frac{1}{\varepsilon} \mathcal{X}_{\omega_{\varepsilon}} V_{\varepsilon} u_{\varepsilon}, \mu>0$ is such that the elliptic problem associated is positive and

$$
F_{\varepsilon}\left(x, u^{\varepsilon}\right)=f_{0}\left(x, u^{\varepsilon}\right)+\frac{1}{\varepsilon} \mathcal{X}_{\omega_{\varepsilon}} g_{0}\left(x, u^{\varepsilon}\right)
$$

where we denote by $\mathcal{X}_{\omega_{\varepsilon}}$ the characteristic function of the set $\omega_{\varepsilon}$. Thus the potential functions and the effective reaction are "concentrated" in $\omega_{\varepsilon}$. Note that we assume that both $f_{0}$ and $g_{0}$ are defined on $\bar{\Omega} \times \mathbb{R}$.

We are interested in the behavior, for small $\varepsilon$, of the attractor of this parabolic problem.

Below we will assume several hypotheses that imply

$$
\frac{1}{\varepsilon} \mathcal{X}_{\omega_{\varepsilon}} V_{\varepsilon} \rightarrow \mathcal{X}_{\Gamma} V_{0}
$$

for some function $V_{0}$ defined on $\Gamma$ and

$$
F_{\varepsilon}(x, u) \rightarrow F_{0}(x, u)=f_{0}(x, u)+\mathcal{X}_{\Gamma} g_{0}(x, u)
$$

in "some sense" (see [4]). So, we consider the limit parabolic problem given by

$$
\left(P_{0}\right) \equiv\left\{\begin{array}{cc}
u_{t}-\Delta u+\mu u=f_{0}(x, u) & \text { in }(0, T) \times \Omega \\
\frac{\partial u}{\partial n}+V_{0} u=g_{0}(x, u) & \text { on } \Gamma \\
u(0)=u_{0} \in H^{1}(\Omega) . &
\end{array}\right.
$$

Our goal is to prove that the family of sets $\mathcal{A}_{\varepsilon}$ global attractor of $\left(P_{\varepsilon}\right)$ is upper semicontinuous at $\varepsilon=0$ in $H^{1}(\Omega)$, that is: $\operatorname{dist}_{H^{1}(\Omega)}\left(\mathcal{A}_{\varepsilon}, \mathcal{A}_{0}\right) \mapsto 0$, if $\varepsilon \mapsto 0$ with

$$
\operatorname{dist}_{H^{1}(\Omega)}\left(\mathcal{A}_{\varepsilon}, \mathcal{A}_{0}\right):=\sup _{u^{\varepsilon} \in \mathcal{A}_{\varepsilon}} \inf _{u^{0} \in \mathcal{A}_{0}}\left\{\left\|u^{\varepsilon}-u^{0}\right\|_{H^{1}(\Omega)}\right\}
$$


where $\mathcal{A}_{0}$ is the global attractor associated to the limit problem (1.2).

In order to prove this result about the global attractor for the parabolic problem, we use some previous results about the concentrating integral and the elliptic problem associated to the parabolic problem, see [4, 5].

\section{Upper Semicontinuity of Attractors}

We consider the family of parabolic problems (1.1), for $\varepsilon \in\left(0, \varepsilon_{0}\right]$

$$
\left(P_{\varepsilon}\right) \equiv\left\{\begin{array}{cc}
u_{t}^{\varepsilon}+A_{\varepsilon} u^{\varepsilon}+\mu u^{\varepsilon}=F_{\varepsilon}\left(x, u^{\varepsilon}\right) & \text { in }(0, T) \times \Omega \\
\frac{\partial u^{\varepsilon}}{\partial n}=0 & \text { on } \Gamma \\
u^{\varepsilon}(0)=u_{0} \in H^{1}(\Omega) &
\end{array}\right.
$$

where $A_{\varepsilon} u^{\varepsilon}=-\Delta u^{\varepsilon}+\frac{1}{\varepsilon} \mathcal{X}_{\omega_{\varepsilon}} V_{\varepsilon} u_{\varepsilon}$, and $F_{\varepsilon}\left(x, u^{\varepsilon}\right)=f_{0}\left(x, u^{\varepsilon}\right)+\frac{1}{\varepsilon} \mathcal{X}_{\omega_{\varepsilon}} g_{0}\left(x, u^{\varepsilon}\right)$.

Throughout this section we will assume that

$$
\frac{1}{\varepsilon} \int_{\omega_{\varepsilon}}\left|V_{\varepsilon}\right|^{\rho} \leq K_{1}
$$

with $\rho>N-1$, and $K_{1}$ a positive constant independent of $\varepsilon$, and that there exists a function $V_{0} \in L^{\rho}(\Gamma)$ such that for any smooth function $\varphi$, we have

$$
\lim _{\varepsilon \rightarrow 0} \frac{1}{\varepsilon} \int_{\omega_{\varepsilon}} V_{\varepsilon} \varphi=\int_{\Gamma} V_{0} \varphi .
$$

Next, we consider the parabolic problem given by

$$
\left(P_{0}\right) \equiv\left\{\begin{array}{cc}
u_{t}-\Delta u+\mu u=f_{0}(x, u) & \text { in }(0, T) \times \Omega \\
\frac{\partial u}{\partial n}+V_{0} u=g_{0}(x, u) & \text { on } \Gamma \\
u(0)=u_{0} \in H^{1}(\Omega) . &
\end{array}\right.
$$

We assume also the the following conditions for the nonlinearity functions $f_{0}, g_{0}$ :

Growth conditions $(G): f_{0}, g_{0}: \bar{\Omega} \times \mathbb{R} \mapsto \mathbb{R}, \varepsilon \in\left[0, \varepsilon_{0}\right]$ are locally Lipschitz uniformly in $x \in \Omega$ and $x \in \bar{\Omega}$ respectively and satisfy the following growth conditions:

If $N>2$ we assume that

$$
|j(x, u)-j(x, v)| \leq c|u-v|\left(|u|^{\sigma-1}+|v|^{\sigma-1}+1\right)
$$

with $j=f_{0}$ or $j=g_{0}$ and with exponents $\sigma_{f}$ and $\sigma_{g}$ respectively, such that

$$
\sigma_{f} \leq \frac{N+2}{N-2} \quad \text { and } \quad \sigma_{g} \leq \frac{N}{N-2}
$$

If $N=2$ we assume that for every $\eta>0$ there exists $c_{\eta}>0$ such that

$$
|j(x, u)-j(x, v)| \leq c_{\eta}|u-v|\left(e^{\eta|u|^{2}}+e^{\eta|v|^{2}}\right) .
$$


These conditions imply the local existence and uniqueness of solutions of (1.1) and (1.2), see J.Arrieta, A. Rodriguez-Bernal et al. 2.

We assume also the following conditions which ensure that local solutions of the nonlinear parabolic problems (1.1) and (1.2) are globally defined and we have well defined semigroups in $H^{1}(\Omega)$,

$$
T_{\varepsilon}(t) u_{0}=u^{\varepsilon}\left(t, x ; u_{0}\right), \quad 0 \leq \varepsilon \leq \varepsilon_{0},
$$

see [3. Note that the nonlinear semigroups are given by the variation of constants formula

$$
T_{\varepsilon}\left(t, u_{0}\right)=e^{-A_{\varepsilon}^{*} t} u_{0}+\int_{0}^{t} e^{-A_{\varepsilon}^{*}(t-s)} F_{\varepsilon}\left(\cdot, T_{\varepsilon}\left(s, u_{0}\right)\right) d s
$$

with $A_{\varepsilon}^{*}=A_{\varepsilon}+\mu I$ and $\varepsilon \in\left[0, \varepsilon_{0}\right)$, see [7].

Sign conditions $(S)$ Assume in addition that there exist $C \in L^{p}(\Omega), 0 \leq D \in L^{p}(\Omega), p>$ $\frac{N}{2}$ and $E \in L^{q}(\Omega), 0 \leq F \in L^{q}(\Omega), q>N-1$ such that

$$
s f_{0}(x, s) \leq C(x) s^{2}+D(x)|s|, \quad x \in \Omega, s \in \mathbb{R}, \quad 0 \leq \varepsilon \leq \varepsilon_{0}
$$

and

$$
s g_{0}(x, s) \leq E(x) s^{2}+F(x)|s|, \quad x \in \bar{\Omega}, s \in \mathbb{R}, \quad 0 \leq \varepsilon \leq \varepsilon_{0} .
$$

Moreover we assume there exist positive constants $K_{i}, i=2,3$ independent of $\varepsilon$ such that

$$
\frac{1}{\varepsilon} \int_{\omega_{\varepsilon}}|E|^{\rho} \leq K_{2}, \quad \text { with } \rho>N-1,
$$

and

$$
\frac{1}{\varepsilon} \int_{\omega_{\varepsilon}}|F|^{r} \leq K_{3}, \quad \text { with } r>\max \left\{1, \frac{2(N-1)}{N}\right\} .
$$

Dissipative condition $(D)$ Finally we assume the first eigenvalue, $\lambda_{1}$, of the following problem is positive

$$
\left(P_{0}^{1}\right) \equiv\left\{\begin{array}{cc}
-\Delta \varphi-C \varphi+\mu \varphi=\lambda_{1} \varphi & \text { in } \Omega \\
\frac{\partial \varphi}{\partial n}+V_{0} \varphi=E \varphi & \text { on } \Gamma .
\end{array}\right.
$$

With these assumptions our goal is to prove the upper semicontinuity of the family of global attractors. In order to prove this, we use the previous result for the elliptic problem (see J.Arrieta, A. Jimenez-Casas, A.Rodriguez-Bernal [4), and the following lemmas.

Lemma 2.1 Under the above hypotheses on $V_{\varepsilon}, f_{0}, g_{0}$, for sufficiently small $0 \leq \varepsilon$, problems (1.1) and (1.2) have global attractors $\mathcal{A}_{\varepsilon}$.

Moreover, there exists $R>0$ independent of $\varepsilon \geq 0$, such that

$$
\sup _{0 \leq \varepsilon \leq \varepsilon_{0}} \sup _{u \in \mathcal{A}_{\varepsilon}}\|u\|_{L^{\infty}(\Omega)} \leq R .
$$


Proof We denote by $\lambda_{1}^{\varepsilon}$ the first eigenvalue of the following elliptic problem

$$
\left\{\begin{array}{c}
-\Delta \phi^{\varepsilon}+\frac{1}{\varepsilon} \mathcal{X}_{\omega_{\varepsilon}}\left(V_{\varepsilon}-E\right) \phi^{\varepsilon}+(\mu-C) \phi^{\varepsilon}=\lambda_{1}^{\varepsilon} \phi \text { on } \Omega \\
\frac{\partial \phi^{\varepsilon}}{\partial n}=0 \text { in } \Gamma .
\end{array}\right.
$$

By the spectral convergence obtained in [4, we have $\lambda_{1}^{\varepsilon} \mapsto \lambda_{1}$ with $\lambda_{1}$ the first eigenvalue of the elliptic limit problem (2.1). Hence for small enough $\varepsilon_{0}$ we have $\lambda_{1}^{\varepsilon}>0$ for every $0 \leq \varepsilon \leq \varepsilon_{0}$.

We split the proof in several steps.

Step 1: Since, $\lambda_{1}^{\varepsilon}>0$, for $0 \leq \varepsilon \leq \varepsilon_{0}$, following the arguments in [3], see also 9], we prove that

$$
\lim \sup _{t \rightarrow \infty}\left|u^{\varepsilon}\left(t, x ; u_{0}\right)\right| \leq\left|\Phi^{\varepsilon}(x)\right|
$$

uniformly in $x \in \bar{\Omega}$ and for $u_{0} \in B$ in a bounded set $B$ in $H^{1}(\Omega)$, where $\Phi^{\varepsilon}(x)$ is the unique solution of

$$
\left\{\begin{array}{c}
-\Delta \Phi^{\varepsilon}+\frac{1}{\varepsilon} \mathcal{X}_{\omega_{\varepsilon}}\left(V_{\varepsilon}-E\right) \Phi^{\varepsilon}+(\mu-C) \Phi^{\varepsilon}=D+\frac{1}{\varepsilon} \mathcal{X}_{\omega_{\varepsilon}} F \text { on } \Omega \\
\frac{\partial \Phi^{\varepsilon}}{\partial n}=0 \text { in } \Gamma .
\end{array}\right.
$$

Step 2: From the convergence results for elliptic problems in [4], we prove that $\Phi^{\varepsilon}(x) \rightarrow$ $\Phi^{0}(x)$, as $\varepsilon \rightarrow 0$, in $\mathcal{C}^{\beta}(\bar{\Omega})$, for some $\beta>0$, where $\Phi^{0}(x)$ the unique solution of the following problem

$$
\left\{\begin{array}{c}
-\Delta \Phi^{0}-(C-\mu) \Phi^{0}=D \text { in } \Omega \\
\frac{\partial \Phi^{0}}{\partial n}+V_{0} \Phi^{0}=E \Phi^{0}+F \text { in } \Gamma .
\end{array}\right.
$$

Thus, from the smoothing effect of the equations and the results in [6] we get that problems (1.1) and (1.2) have global compact attractors $\mathcal{A}_{\varepsilon}$ in $H^{1}(\Omega)$, for $0 \leq \varepsilon \leq \varepsilon_{0}$. Also, there exists $R$ independent of $\varepsilon>0$ and $\varepsilon_{0}$ enough small, such that

$$
\sup _{0 \leq \varepsilon \leq \varepsilon_{0}} \sup _{u \in \mathcal{A}_{\varepsilon}}\|u\|_{L^{\infty}(\Omega)} \leq R_{\text {. }}
$$

With this and the variation of constants formula we get

Lemma 2.2 Under the above hypotheses on $V_{\varepsilon}, f_{0}, g_{0}$ we have that, there exists $R>0$ such that

$$
\sup _{0 \leq \varepsilon \leq \varepsilon_{0}} \sup _{u \in \mathcal{A}_{\varepsilon}}\|u\|_{H^{1}(\Omega) \cap L^{\infty}(\Omega)} \leq R .
$$

In particular, $\mathcal{A}_{0}$ attracts $\cup_{\varepsilon \in\left(0, \varepsilon_{0}\right)} \mathcal{A}_{\varepsilon}$ in $H^{1}(\Omega)$.

The following Lemma 2.3 proved in 8 , shows several technical results on the behavior of concentrating function as $\varepsilon \mapsto 0$. This will allow us to prove the convergence of the nonlinearities $F_{\varepsilon}$ given by the Lemma 2.4. Note that below we make use of the intermediate sobolev spaces $H^{s}(\Omega)$ and their dual spaces which we denote $H^{-s}(\Omega)$ for $\frac{1}{2} \leq s \leq 1$, that is $H^{-s}(\Omega) \equiv\left(H^{s}(\Omega)\right)^{\prime}$. 
Lemma 2.3 Under the above hypotheses, if $\|v\|_{H^{1}(\Omega) \cap L^{\infty}(\Omega)} \leq R$, then we have that:

i) For any $\frac{1}{2}<s \leq 1$ there exists $M(R)$ a positive constant independent of $\varepsilon$ such that for any smooth function $\varphi$ up to the boundary of $\Omega$,

$$
\left|\frac{1}{\varepsilon} \int_{\omega_{\varepsilon}} g_{0}(v) \varphi\right| \leq M(R)\|\varphi\|_{H^{s}(\Omega)} .
$$

ii) There exists $M(\varepsilon, R) \mapsto 0$ if $\varepsilon \mapsto 0$ such that for any smooth function $\varphi$ up to the boundary of $\Omega$,

$$
\left|\frac{1}{\varepsilon} \int_{\omega \varepsilon} g_{0}(v) \varphi-\int_{\Gamma} g_{0}(v) \varphi\right| \leq M(\varepsilon, R)\|\varphi\|_{H^{1}(\Omega)} .
$$

Proof The proof of this Lemma can be found in [8].

With this, we get the following result that states the convergence of the nonlinear terms of the problems.

Lemma 2.4 Under the above hypotheses we have that for any $\frac{1}{2}<s<1$ :

i) There exists $C>0$ independent of $\varepsilon>0$ such that

$$
\sup _{v \in \mathcal{A}_{\varepsilon}}\left\{\left\|F_{\varepsilon}(v)\right\|_{H^{-s}(\Omega)},\left\|F_{0}(v)\right\|_{H^{-s}(\Omega)}\right\} \leq C .
$$

ii) There exists $M(\varepsilon)$ with $M(\varepsilon) \mapsto 0$ if $\varepsilon \mapsto 0$, such that

$$
\sup _{v \in \mathcal{A}_{\varepsilon}}\left\|F_{\varepsilon}(v)-F_{0}(v)\right\|_{H^{-s}(\Omega)} \leq M(\varepsilon) .
$$

Proof Part i) follows from Lemmas 2.2 and 2.3 i). For part ii), note that from Lemmas 2.1 and 2.4, we obtain that:

$$
\sup _{v \in \mathcal{A}_{\varepsilon}}\left\|F_{\varepsilon}(v)-F_{0}(v)\right\|_{H^{-1}(\Omega)} \leq M(\varepsilon) \rightarrow 0
$$

as $\varepsilon \rightarrow 0$.

Now, fix $\frac{1}{2}<s_{0}<1$. Then for any $s$ such that $-\frac{1}{2}<-s_{0}<-s<-1$, by interpolation

$$
\left\|F_{\varepsilon}(v)-F_{0}(v)\right\|_{H^{-s}(\Omega)} \leq\left\|F_{\varepsilon}(v)-F_{0}(v)\right\|_{H^{-s_{0}(\Omega)}}^{\theta}\left\|F_{\varepsilon}(v)-F_{0}(v)\right\|_{H^{-1}(\Omega)}^{1-\theta}
$$

for some $0<\theta<1$. Again by Lemmas 2.2 and $2.3 \mathrm{i}$ ), the first term in the right hand side above is bounded while the second goes to zero, both uniformly for $v \in \mathcal{A}_{\varepsilon}$, and we conclude.

On the other hand, from the spectral convergence of the linear operators we get, see [1] for a similar result.

Lemma 2.5 Under the above hypotheses, let $\frac{1}{2}<s<1$. Then, there exist $\alpha \in\left(\frac{1+s}{2}, 1\right)$ and a function $C_{0}(\varepsilon) \geq 0$ with $C_{0}(\varepsilon) \mapsto 0$ if $\varepsilon \mapsto 0$, such that for all $h \in H^{-s}(\Omega)$ we have that

$$
\left\|e^{-A_{\varepsilon}^{*} t} h-e^{-A_{0}^{*} t} h\right\|_{H^{1}(\Omega)} \leq C_{0}(\varepsilon) t^{-\alpha}\|h\|_{H^{-s}(\Omega)}, \quad t>0 .
$$


With all the above we can then obtain the convergence of the nonlinear semigroups.

Lemma 2.6 Under the above hypothesis let $\frac{1}{2}<s_{0}<1$ and some fixed $\tau>0$. Then, there exists a function $C(\varepsilon) \geq 0$ with $C(\varepsilon) \mapsto 0$ if $\varepsilon \mapsto 0$, such that for $u_{\varepsilon} \in \mathcal{A}_{\varepsilon}, \varepsilon \in\left(0, \varepsilon_{0}\right)$,

$$
\left\|T_{\varepsilon}\left(t, u_{\varepsilon}\right)-T_{0}\left(t, u_{\varepsilon}\right)\right\|_{H^{1}(\Omega)} \leq M(\tau) C(\varepsilon) t^{-\alpha} \quad \text { for } t \in(0, \tau]
$$

for some $\alpha \in\left(\frac{1+s_{0}}{2}, 1\right)$.

Proof We consider the nonlinear semigroup given by the variation of constant formula:

$$
T_{\varepsilon}\left(t, u_{\varepsilon}\right)=e^{-A_{\varepsilon}^{*} t} u_{\varepsilon}+\int_{0}^{t} e^{-A_{\varepsilon}^{*}(t-s)} F_{\varepsilon}\left(x, T_{\varepsilon}\left(s, u_{\varepsilon}\right)\right) d s
$$

where $A_{\varepsilon}^{*}=A_{\varepsilon}+\mu I, \varepsilon \in\left[0, \varepsilon_{0}\right]$ and

$$
T_{0}\left(t, u_{\varepsilon}\right)=e^{-A_{0}^{*} t} u_{\varepsilon}+\int_{0}^{t} e^{-A_{0}^{*}(t-s)} F_{0}\left(x, T_{0}\left(s, u_{\varepsilon}\right)\right) d s .
$$

From (2.2) and (2.3), together with the previous results, we will get below that

$$
\begin{gathered}
\left\|T_{\varepsilon}\left(t, u_{\varepsilon}\right)-T_{0}\left(t, u_{\varepsilon}\right)\right\|_{H^{1}(\Omega)} \leq M^{*} C(\varepsilon) t^{-\alpha}+ \\
+M^{*} \int_{0}^{t}(t-s)^{-\alpha}\left\|T_{\varepsilon}\left(s, u_{\varepsilon}\right)-T_{0}\left(s, u_{\varepsilon}\right)\right\|_{H^{1}(\Omega)} d s
\end{gathered}
$$

for some $M^{*}$ depending on $\tau$. Hence, applying the singular Gronwall Lemma, Lemma 7.1.1 in [7, to (2.4), we get the result.

We now split the proof of (2.4) in several steps. In effect, from (2.2) and (2.3) we have that:

$$
\begin{gathered}
\left\|T_{\varepsilon}\left(t, u_{\varepsilon}\right)-T_{0}\left(t, u_{\varepsilon}\right)\right\|_{H^{1}(\Omega)} \leq\left\|e^{-A_{\varepsilon}^{*} t} u_{\varepsilon}-e^{-A_{0}^{*} t} u_{\varepsilon}\right\|_{H^{1}(\Omega)}+ \\
+\int_{0}^{t}\left\|e^{-A_{\varepsilon}^{*}(t-s)} F_{\varepsilon}\left(x, T_{\varepsilon}\left(s, u_{\varepsilon}\right)\right)-e^{-A_{0}^{*}(t-s)} F_{\varepsilon}\left(x, T_{\varepsilon}\left(s, u_{\varepsilon}\right)\right)\right\|_{H^{1}(\Omega)} d s+ \\
\quad+\int_{0}^{t}\left\|e^{-A_{0}^{*}(t-s)}\left[F_{\varepsilon}\left(x, T_{\varepsilon}\left(s, u_{\varepsilon}\right)\right)-F_{0}\left(x, T_{\varepsilon}\left(s, u_{\varepsilon}\right)\right)\right]\right\|_{H^{1}(\Omega)} d s+ \\
+\int_{0}^{t}\left\|e^{-A_{0}^{*}(t-s)}\left[F_{0}\left(x, T_{\varepsilon}\left(s, u_{\varepsilon}\right)\right)-F_{0}\left(x, T_{0}\left(s, u_{\varepsilon}\right)\right)\right]\right\|_{H^{1}(\Omega)} d s=I_{1}+I_{2}+I_{3}+I_{4} .
\end{gathered}
$$

Step 1.- From Lemma 2.5 together with Lemma 2.2. we obtain:

$$
I_{1}=\left\|e^{-A_{\varepsilon}^{*} t} u_{\varepsilon}-e^{-A_{0} t} u_{\varepsilon}\right\|_{H^{1}(\Omega)} \leq C_{0}(\varepsilon) t^{-\alpha}\left\|u_{\varepsilon}\right\|_{H^{-s_{0}(\Omega)}} \leq C_{0}(\varepsilon) t^{-\alpha} K_{0}
$$

with $0<C_{0}(\varepsilon) \mapsto 0$ if $\varepsilon \mapsto 0$ and $K_{0}$ a positive constant independent of $\varepsilon$.

Step 2.- Again Lemma 2.5 gives

$$
I_{2}=\int_{0}^{t}\left\|e^{-A_{\varepsilon}^{*}(t-s)} F_{\varepsilon}\left(x, T_{\varepsilon}\left(s, u_{\varepsilon}\right)\right)-e^{-A_{0}^{*}(t-s)} F_{\varepsilon}\left(x, T_{\varepsilon}\left(s, u_{\varepsilon}\right)\right)\right\|_{H^{1}(\Omega)} d s \leq
$$




$$
\leq C_{0}(\varepsilon) \int_{0}^{t}(t-s)^{-\alpha}\left\|F_{\varepsilon}\left(x, T_{\varepsilon}\left(s, u_{\varepsilon}\right)\right)\right\|_{H^{-s_{0}(\Omega)}} d s .
$$

Now, from Lemma 2.4 and using thet the attractor $\mathcal{A}_{\varepsilon}$ is invariant for the semigroup $T_{\varepsilon}$, we obtain a positive constant $K_{1}$ independent of $\varepsilon$ such that $\left\|F_{\varepsilon}\left(\cdot, T_{\varepsilon}\left(s, u_{\varepsilon}\right)\right)\right\|_{H^{-s_{0}(\Omega)}} \leq K_{1}$. From this

$$
I_{2} \leq C_{0}(\varepsilon) \frac{K_{1}}{1-\alpha} t^{1-\alpha} \leq C_{0}(\varepsilon) K_{2} t^{-\alpha}
$$

since $t \leq \tau$.

Step 3.-

$$
\begin{aligned}
& I_{3}=\int_{0}^{t} \| e^{-A_{0}^{*}(t-s)}\left(F_{\varepsilon}\left(x, T_{\varepsilon}\left(s, u_{\varepsilon}\right)\right)-F_{0}\left(x, T_{\varepsilon}\left(s, u_{\varepsilon}\right)\right) \|_{H^{1}(\Omega)} d s \leq\right. \\
& \leq K_{2} \int_{0}^{t}(t-s)^{-\alpha} \| F_{\varepsilon}\left(\left(x, T_{\varepsilon}\left(s, u_{\varepsilon}\right)-F_{0}\left(x, T_{\varepsilon}\left(s, u_{\varepsilon}\right)\right) \|_{H^{-s_{0}(\Omega)}} d s .\right.\right.
\end{aligned}
$$

Using again Lemma 2.4 and the invariance of the attractor, we obtain that $\| F_{\varepsilon}\left(\cdot, T_{\varepsilon}\left(s, u_{\varepsilon}\right)\right)-$ $F_{0}\left(\cdot, T_{\varepsilon}\left(s, u_{\varepsilon}\right)\right) \|_{H^{-s_{0}(\Omega)}} \leq M(\varepsilon)$, with $M(\varepsilon) \mapsto 0$ if $\varepsilon \mapsto 0$ and $I_{3} \leq M(\varepsilon) K_{3} t^{-\alpha}$, since $t \leq \tau$, with $K_{3}$ a positive constant independent of $\varepsilon$ and depending on $\tau$.

Step 4.-

$$
\begin{aligned}
& I_{4}=\int_{0}^{t}\left\|e^{-A_{0}^{*}(t-s)}\left(F_{0}\left(x, T_{\varepsilon}\left(s, u_{\varepsilon}\right)\right)-F_{0}\left(x, T_{0}\left(s, u_{\varepsilon}\right)\right)\right)\right\|_{H^{1}(\Omega)} d s \leq \\
& \leq K_{2} \int_{0}^{t}(t-s)^{-\alpha}\left\|F_{0}\left(x, T_{\varepsilon}\left(s, u_{\varepsilon}\right)\right)-F_{0}\left(x, T_{0}\left(s, u_{\varepsilon}\right)\right)\right\|_{H^{-s_{0}}(\Omega)} d s .
\end{aligned}
$$

Now, from the bounds in Lemma 2.2 and the regularity of the nonlinear terms $f_{0}$ and $g_{0}$ we get that $\left\|F_{0}(u)-F_{0}(v)\right\|_{H^{-s_{0}(\Omega)}} \leq L\|u-v\|_{H^{1}(\Omega)}$ with $L=L(R)$ if the norm of both $u$ and $v$ in $H^{1}(\Omega) \cap L^{\infty}(\Omega)$ is bounded by $R$.

Therefore, we get $I_{4} \leq K_{2} L \int_{0}^{t}(t-s)^{-\alpha}\left\|T_{\varepsilon}\left(s, u_{\varepsilon}\right)-T_{0}\left(s, u_{\varepsilon}\right)\right\|_{H^{1}(\Omega)} d s$.

Puting all the estimates above together, we get (2.4) and the proof is complete. $\square$

Theorem 2.7 Under the above hypothesis about $V_{\varepsilon}, f_{0}, g_{0}$ the family of global attractors of $\left(P_{\varepsilon}\right), \mathcal{A}_{\varepsilon}$, is upper semicontinuous at $\varepsilon=0$ in $H^{1}(\Omega)$, that is:

$$
\operatorname{dist}_{H^{1}(\Omega)}\left(\mathcal{A}_{\varepsilon}, \mathcal{A}_{0}\right) \mapsto 0 \text {, if } \varepsilon \mapsto 0
$$

where

$$
\operatorname{dist}_{H^{1}(\Omega)}\left(\mathcal{A}_{\varepsilon}, \mathcal{A}_{0}\right):=\sup _{u_{\varepsilon} \in \mathcal{A}_{\varepsilon}} \inf _{u_{0} \in \mathcal{A}_{0}}\left\{\left\|u_{\varepsilon}-u_{0}\right\|_{H^{1}(\Omega)}\right\}
$$

Proof In effect, from Lemma 2.2, $\mathcal{A}_{0}$ attracts $\cup_{0<\varepsilon \leq \varepsilon_{0}} \mathcal{A}_{\varepsilon}$, since the latter is a bounded set in $H^{1}(\Omega)$. Hence, given $\delta>0$, there exists $\tau=\tau(\delta)$ such that dist $H_{H^{1}}\left(T_{0}(\tau) u_{\varepsilon}, \mathcal{A}_{0}\right) \leq \frac{\delta}{2}$ for every $u_{\varepsilon} \in \mathcal{A}_{\varepsilon}$ with $\varepsilon \in\left(0, \varepsilon_{0}\right)$.

Next, using that $\mathcal{A}_{\varepsilon}$ is invariant, given $v_{\varepsilon} \in \mathcal{A}_{\varepsilon}$, there exists $u_{\varepsilon}$ such that $T_{\varepsilon}(\tau) u_{\varepsilon}=v_{\varepsilon}$. Therefore,

$$
\operatorname{dist}_{H^{1}}\left(v_{\varepsilon}, \mathcal{A}_{0}\right) \leq\left\|v_{\varepsilon}-T_{0}(\tau) u_{\varepsilon}\right\|_{H^{1}(\Omega)}+\operatorname{dist}_{H^{1}}\left(T_{0}(\tau) u_{\varepsilon}, \mathcal{A}_{0}\right)
$$


Then from Lemma 2.6 it is clear that if $\varepsilon$ is small enough we get

$$
\left\|v_{\varepsilon}-T_{0}(\tau) u_{\varepsilon}\right\|_{H^{1}(\Omega)}=\left\|T_{\varepsilon}(\tau) u_{\varepsilon}-T_{0}(\tau) u_{\varepsilon}\right\|_{H^{1}(\Omega)} \leq \frac{\delta}{2},
$$

and we conclude.

\section{References}

[1] J.M. Arrieta, "Spectral Behavior and Uppersemicontinuity of Attractors" International Conference on Differential Equations (EQUADIFF'99) Berlin. World Scientific, 615-621 (2000).

[2] J.M. Arrieta, A. N. Carvalho, A. Rodríguez-Bernal, "Parabolic problems with nonlinear boundary conditions and critical nonlinearities", J. Differential Equations 156, 376-406 (1999).

[3] J.M. Arrieta, A. N. Carvalho, A. Rodríguez-Bernal, "Attractors of Parabolic Problems with Critical Nonlinearities. Uniform Bounds". Comm.P.D.E.'s 25, vol 1-2, 1-37 (2000).

[4] J.M. Arrieta, A. Jiménez-Casas, A. Rodríguez-Bernal "Nonhomogeneous flux condition as limit of concentrated reactions ", Revista Iberoamericana de Matematicas vol 24, no 1, 183-211 (2008).

[5] J.M.Arrieta, A. Rodríguez-Bernal, J. Rossi, "The best Sobolev trace constant as limit of the usual Sobolev constant for small strips near the boundary". Proceeding of the Royal Society of Edinburgh. Section A. Mathematics, vol 138A, 223-237 (2006).

[6] J.K. Hale, "Asymptotic Behavior of Dissipative System", American Mathematical Society (1988).

[7] D. Henry, "Geometry Theory of Semilinear Parabolic Equations", Springer-Verlag, (1981).

[8] A. Jiménez-Casas, A. Rodríguez-Bernal "Upper Semicontinuity of Attractors for Parabolic Problem with terms concentrating on the boundary", in preparation.

[9] A. Rodríguez-Bernal, A. Vidal-López "Extremal equilibria for nonlinear parabolic equations in bounded domains and applications", Journal of Differential Equations 244, 2983-3030 (2008). 